\title{
Investigation of Magnetite Thin-Films Produced by Pulsed Laser Deposition
}

\author{
R.A. Lindley, S.P. Sena, H.J. Blythe and G.A. Gehring \\ Department of Physics, The University of Sheffield, Sheffield S3 7RH, U.K.
}

\begin{abstract}
Thin-films of magnetite, $\mathrm{Fe}_{3} \mathrm{O}_{4}$, have been produced by the $\mathrm{XeCl}$ excimer laser ablation of both $\mathrm{Fe}_{3} \mathrm{O}_{4}$ and metallic $\mathrm{Fe}$ targets. The ablation of metallic $\mathrm{Fe}$ has been shown to produce about two orders of magnitude smaller concentration of boulders than the ablation of bulk magnetite. The use of single-crystal, lattice-matching substrates was found to be necessary at lower fluences $\left(1-3 \mathrm{~J} \mathrm{~cm}^{-2}\right)$ for the ablation of metallic $\mathrm{Fe}$, whereas polycrystalline, non-lattice-matching substrates were adequate for deposition with moderate laser fluences $\left(5-6 \mathrm{~J} \mathrm{~cm}^{-2}\right)$ Film quality, which has been assessed by examining the Verwey transition via electrical conductivity and magnetic measurements, was found to decrease with decreasing film thickness.
\end{abstract}

\section{INTRODUCTION}

Although bulk magnetite has been extensively studied over many years, magnetite thin-films have recently received considerable attention on account of their potential for industrial applications: magnetite thin-films can be oxidised to produce $\gamma-\mathrm{Fe}_{2} \mathrm{O}_{3}$ for use as a magnetic recording medium and magnetic multilayers involving magnetite can be used to fabricate thin-film systems with desirable magnetic properties. A range of techniques has been used for the production of high quality magnetite thin-films and multilayers including pulsed laser deposition (PLD) [1 - 3]. In most cases of PLD, the target has been of the same composition as that of the resulting thin-film. However, very recently, Kennedy [4] has reported on thin-film magnetite produced by the ablation of metallic $\mathrm{Fe}$. In the present work, magnetite thin-films have been produced using the technique of PLD with either a bulk magnetite target or a metallic Fe target. Electrical conductivity and magnetic measurements were made to determine the Verwey transition, which was used an indication of film quality.

\section{EXPERIMENTAL DETAILS}

Magnetite thin-films were produced by the ablation of bulk magnetite using a range of laser fluences $\left(0.4-6 \mathrm{~J} \mathrm{~cm}^{-2}\right)$ and varying other deposition conditions. The films were deposited onto polycrystalline quartz and Corning Glass substrates. However, thin-films made in this fashion were found to have poor reproducibility. X-ray diffraction revealed that the magnetite target changed composition following successive deposition runs performed in a range of oxygen pressures and showed the presence of small amounts of haematite

Films were also made by the ablation of metallic $\mathrm{Fe}$ (natural and enriched) onto polycrystalline quartz substrates using moderate fluences $\left(5.4-5.7 \mathrm{~J} \mathrm{~cm}^{-2}\right)$ in $4 \mathrm{mTorr}$ of oxygen and with no direct substrate heating. Thin-films made in this fashion were found to have good reproducibility except for the c-axis orientation of the polycrystalline thin-films, which varied noticeably with substrate temperature. However, extensive attempts to deposit magnetite thin-films onto polycrystalline quartz substrates using lower fluences $\left(1-3 \mathrm{~J} \mathrm{~cm}^{-2}\right)$ in the manner of Masterson et al. [2] were unsuccessful. Magnetite thin-films were made by the ablation of metallic $\mathrm{Fe}$ onto single crystal substrates $\mathrm{MgO}(100)$ and $\mathrm{Al}_{2} \mathrm{O}_{3}(0001)$ using low fluences $\left(2.1-2.5 \mathrm{~J} \mathrm{~cm}^{-2}\right)$ in a $0.1 \mathrm{mTorr}$ partial pressure of oxygen and at a nominal substrate temperature of $300^{\circ} \mathrm{C}$, although this value is strongly influenced by the proximity of the plume. Immediately after each deposition, the films were cooled at a rate of about $9 \mathrm{~K} \mathrm{~min}^{-1}$. X-ray diffraction revealed that the resulting film c-axis orientations were $(100)$ and (111), respectively. These single-crystal substrates were chosen because they are nearly lattice matching to the magnetite thin films, the oxygen-oxygen separations differing by only $0.3 \%$ for the $\mathrm{MgO}$ substrates and $8 \%$ for the $\mathrm{Al}_{2} \mathrm{O}_{3}$ substrates. Film thickness was determined by either breaking the substrate and viewing the thin-film edge-on with a scanning electron microscope or half-covering an additional substrate with Kapton and measuring the resulting film-step with a Talystep thickness gauge. The d.c. conductivities of the thin-films were measured using a standard four-point, van der Paaw configuration. Magnetic measurements were made using a SQUID magnetometer. 

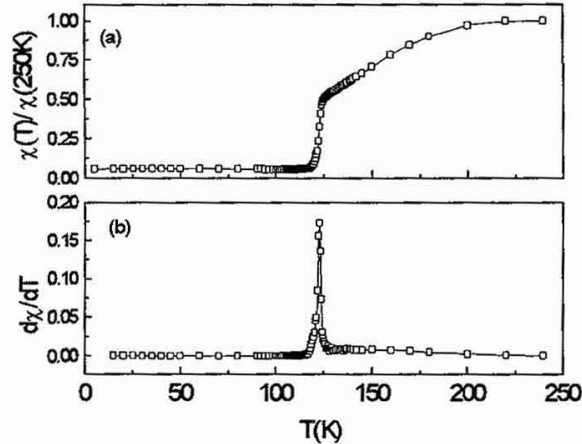

Fig.1 (a) Temperature dependence of the normalized susceptibility of a $1030 \mathrm{~nm}$ magnetite film measured in a field of $50 \mathrm{mT}$.

(b) Derivative of (a).

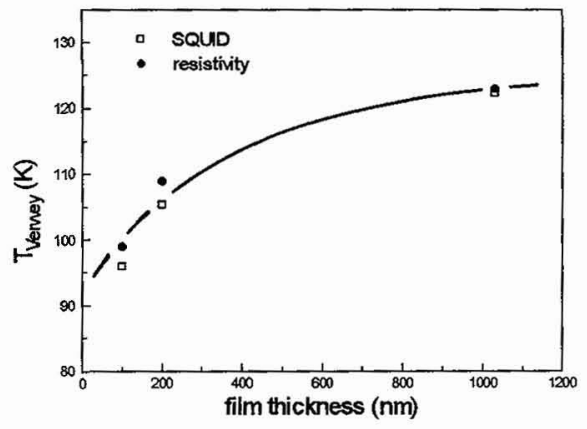

Fig. 2 Variation of Verwey temperature as a function of filmthickness determined from magnetic and electrical resistivity measurements. The line is merely a guide for the eye.

\section{RESULTS AND DISCUSSION}

Comparison of a number of thin-films produced by the PLD of magnetite and metallic Fe targets shows that the ablation of the magnetite target produced approximately $4000-6000$ boulders $\mathrm{mm}^{-2}$ whereas the ablation of the metallic Fe target produced approximately $20-80$ boulders $\mathrm{mm}^{-2}$, i.e., a reduction of about two orders of magnitude. In addition, the use of metallic $\mathrm{Fe}$ is advantageous because the target constituency is stable, target heating is beneficial and defect density is less important to the production of boulders than with the ablation of magnetite.

Fig. I(a) shows the temperature dependence, as measured in a field of $50 \mathrm{mT}$, of the magnetic moment of a $1030 \mathrm{~nm}$ thick film which had been grown on a $5 \times 5 \mathrm{~mm} \mathrm{MgO} \mathrm{(100)} \mathrm{substrate} \mathrm{by} \mathrm{the} \mathrm{ablation} \mathrm{of} \mathrm{metallic} \mathrm{Fe}$. In order to determine the position of the Verwey temperature, in fig.l(b), we have plotted the derivative of this curve. This yields a Verwey temperature of $123 \mathrm{~K}$, a value very close to that of good quality bulk, single-crystal material.

Fig.2 shows the dependence of Verwey temperature on film thickness. There is reduction of Verwey temperature to lower temperatures with decreasing film thickness and, in the case of the $50 \mathrm{~nm}$ film, the transition is completely suppressed. This behaviour is also confirmed by measurements of the electrical resistivity of the same films when, once again, the $50 \mathrm{~nm}$ film exhibited no Verwey transition.

Our interpretation of the dependence of the Verwey transition on film thickness is based on the assumption that interfacial strain induced in the epitaxial film plays an increasing role as film thickness decreases such that, in the thinnest film produced, the lattice mis-match is sufficient to eliminate the transition; the sensitivity of the transition temperature to filmsubstrate strain is well-established [5]. With increasing film thickness, the influence of strain decreases until, for the 1030 $\mathrm{nm}$ film, the Verwey temperature has attained the value associated with bulk material. It is interesting that the values of the transition temperatures determined by both electrical and magnetic measurements yield essentially the same values, despite the fact that resistivity measurements are probably more sensitive to film quality than the magnetic measurements

A second set of films produced by the ablation of ${ }^{57} \mathrm{Fe}$ also exhibit a reduction of Verwey temperature with decreasing film thickness. These samples were investigated at room temperature using conversion electron Mössbauer spectroscopy; preliminary results indicate that the samples contained significant amounts of both metallic $\mathrm{Fe}$ and $\mathrm{Fe}$ in an, as yet, unspecified paramagnetic phase. At the present time, it seems feasible that this paramagnetic phase may result from an interdiffusion of the $\mathrm{Fe}$ and $\mathrm{Mg}$ in the vicinity of the substrate - film interface due to the high substrate temperature during deposition. In view of this, it seems probable that the thin-films produced from natural $\mathrm{Fe}$ also contained similar impurities. A more detailed investigation of this effect will be published at a later date [6].

\section{Acknowledgements}

We are grateful to Dr. Ch. Sauer who performed the Mössbauer spectroscopy measurements on the ${ }^{57} \mathrm{Fe}_{3} \mathrm{O}_{4}$ samples.

\section{References}

[1] Kleint C.A., Semmelhack H.C., Lorenz M. and Krause M.K., J. Mag. and Mag. Mat. 144 (1995) 725

[2] Masterson H.J., Lunney J.C., Coey J.M.D. and Moukarika A, J. Mag. and Mag. Mat. 115 (1992) 155

[3] Joshi S., Nawathey R., Koinkar V.N., Godbole V.P., Chaudhari S.M., Ogale S.B. and Date S.K., J.Appl.Phys., 64, (1988) 5647

[4] Kennedy R.J., IEEE Transactions on Magnetics, Vol. 31, (1995) No.6, 3829

[5] Berry S.D., Lind D.M., Chern G. and Mathias H., J. Mag. and Mag. Mat. 123, (1993) 126

[6] Blythe H.J., Gehring G.A., Lindley R.A., Sauer Ch. and Sena S.P.: to be published. 